\title{
Analysis of NuSTAR and Suzaku observations of Cyg X-1 in the hard state: evidence for a truncated disc geometry
}

\author{
Rupal Basak,${ }^{1 \star}$, Andrzej A. Zdziarski,${ }^{1 \star}{ }^{\star}$ Michael Parker ${ }^{2 \star}$ and Nazma Islam ${ }^{1 \star}$ \\ ${ }^{1}$ Nicolaus Copernicus Astronomical Center, Polish Academy of Sciences, Bartycka 18, PL-00-716 Warszawa, Poland \\ ${ }^{2}$ Institute of Astronomy, Madingley Road, Cambridge, CB3 OHA, UK
}

Submitted 2017 May 18

\begin{abstract}
The geometry of the accretion flow in black-hole X-ray binaries in the hard state, in particular the position of the disc inner edge, has been a subject of intense debate in recent years. We address this issue by performing a spectral study of simultaneous observations of Cyg X-1 in the hard state by NuSTAR and Suzaku. The same data were analysed before, and modelled by a lamppost containing hybrid electrons and located very close to the horizon, which emission was incident on a surrounding disc extending almost to the innermost stable circular orbit. We re-analyse the incident continuum model and show that it suffers from the lack of physical self-consistency. Still, the good fit to the data provided by this model indicates that the real continuum has a similar shape. We find it features a strong soft X-ray excess below a few $\mathrm{keV}$, which we model as a soft thermal-Comptonization component, in addition to the main hard thermal-Compton component. This continuum model with reflection of both components yields the overall lowest $\chi^{2}$ and has a geometry with a hot inner accretion flow and a disc truncated at $\simeq 13-20$ gravitational radii. On the other hand, we have also found a spectral solution with a lamppost at a large height and a disc that can extend to the innnermost stable circular orbit. Overall, we find the fitted truncation radius depends on the assumed continuum and geometry.
\end{abstract}

Key words: accretion, accretion discs - black hole physics - stars: individual: Cyg X-1 $\mathrm{X}$-rays: binaries - X-rays: individual: Cyg X-1

\section{INTRODUCTION}

Determination of the geometry of inner regions of accreting blackhole $(\mathrm{BH})$ sources is of major importance for our understanding of the physics of those sources. There exist a large number of diverse models of the X-ray emission of those sources, explaining it in terms of accretion discs and their coronae, hot inflows and outflows, and jets. The two main types of those sources are active galactic nuclei and accreting X-ray binaries (XRBs) containing BHs. Here, we concentrate on the latter.

Luminous BH XRBs show two main spectral states, hard and soft. There is a relative consensus regarding the geometry in the soft state. The main component of the accretion flow is an optically-thick and geometrically-thin accretion disc (Shakura \& Sunyaev 1973; Novikov \& Thorne 1973) extending down to the innermost stable circular orbit (ISCO). The hard X-ray tails present in this state with various relative amplitude are probably due to coronal emission containing hybrid, i.e., both thermal and nonthermal, electrons, Compton scattering disc blackbody photons, e.g., Gierliński et al. (1999), Poutanen \& Vurm (2009).

^rupal@camk.edu.pl, aaz@camk.edu.pl, mlparker@ast.cam.ac.uk, nislam@camk.edu.pl
On the other hand, there has been an ongoing dispute regarding the nature of the hard state. The source has to be capable of emitting hard X-rays, which dominate the energetics in this state. A likely radiative process is Compton scattering by a predominantly thermal plasma, given an excellent description of the hard X-ray spectra by it, e.g., Gierliński et al. (1997). A lot of work has been devoted to the model in which this plasma forms a hot accretion flow surrounded by a cold outer disc, e.g., Shapiro, Lightman \& Eardley (1976), Narayan \& Yi (1995), Done, Gierliński \& Kubota (2007), Yuan \& Narayan (2014), and many other papers. Such a geometry has to be present in quiescence of BH transients and in initial stages of their outbursts (Lasota, Narayan \& Yi 1996). In this model, it is natural to associate the transition to the soft state during a transient outburst with the viscous outer disc reaching the ISCO. This model does explain a lot of phenomena observed in BH binaries, e.g., correlations between the X-ray power-law index with the relative strength of Compton reflection, the broadening of the $\mathrm{Fe} \mathrm{K} \alpha$ line, the characteristic frequencies in the power spectra, and the luminosity, e.g., Done et al. (2007), Gilfanov (2010), Gierliński, Zdziarski \& Done (2011). Also, the main current model for the lowfrequency QPOs/breaks in the power spectra is the Lense-Thirring precession of an inner hot flow surrounded by an outer disc (e.g., 
Ingram, Done \& Fragile 2009; Ingram \& Done 2011), which requires a disc truncation.

However, this model has been questioned in a large number of papers claiming detections of reflection features being as broad as requiring the disc to extend down to the ISCO (or almost to it) also in luminous hard states. Initially, the hard X-ray emission was modelled in those works by a corona above the disc with a prescribed radial emissivity profile of the reflected component, usually steeply rising toward the ISCO (e.g., Miller et al. 2006; Rykoff et al. 2007; Tomsick et al. 2008). Later, the corona was replaced by a static point-like source on the rotation axis of the $\mathrm{BH}$, so-called lamppost. Martocchia \& Matt (1996), who introduced this model, noted that there was no reason for all emitting particles to be on the symmetry axis and static, but they used that assumption since it greatly simplified the calculation of the disc irradiation. Fit results using the implementation of this model by Dauser et al. (2010) often resulted in extreme parameters location of the lamppost, almost on the horizon at the best fit, e.g., Parker et al. (2015), hereafter P15, in the case of Cyg X-1.

These results have been controversial. In a number of cases, the same observations as those used by the authors claiming the disc extending to the ISCO have been fitted by others, who found the disc to be truncated (Done \& Diaz Trigo 2010; Kolehmainen, Done \& Díaz Trigo 2014; Plant et al. 2015; Basak \& Zdziarski 2016). The differences have been explained by both instrumental effects (in particular, the instrumental pileup) and differences in modelling. In the case of the BH binary GX 339-4, a detailed comparison of different model results is given in Basak \& Zdziarski (2016). In the case of the hard state of Cyg X-1, some works found a truncated disc (Done \& Zycki 1999; Di Salvo et al. 2001; Frontera et al. 2001; Makishima et al. 2008; Yamada et al. 2013), and some, a disc extending to the ISCO (Reis, Fabian \& Miller 2010; Miller, Pooley, Fabian, Nowak, Reis, Cackett, Pottschmidt, \& Wilms 2012; P15).

For the lamppost geometry, Niedźwiecki, Zdziarski \& Szanecki (2016) have pointed out two major problems with its physical self-consistency in the case of the lamppost location close to the BH horizon. Due to light bending, only very few of the emitted photons are able to go to the observer; most either cross the horizon or hit the surrounding disc. This means the actual source luminosity (as measured at infinity) is much larger than that observed. In the case of the Cyg X-1 results of P15, this implies that the accretion rate in the hard state has to be substantially higher than that in the soft state, which is impossible. Furthermore, the source luminosity as locally measured is even higher, due to the additional effects of time dilation and energy shift, which then implies that the source is out of equilibrium between production of $\mathrm{e}^{ \pm}$pairs and their annihilation.

Furthermore, the nature of the transition between the hard and soft states becomes difficult to understand if the disc extends to the ISCO in both of them. The disc component in the hard state has a low maximum temperature, $k T_{\mathrm{bb}} \sim 0.1-0.2 \mathrm{keV}$, and its amplitude is weak, with the Comptonization luminosity being typically a factor of $\sim 10$ higher than that in the disc blackbody. This also means that the accretion rate through the disc in the hard state is much less than the total one. The state transition would then correspond to the disappearance of the hot plasma and an increase of the disc accretion rate by a factor of a few tens (accounting also for the increase of the luminosity in the soft state) caused by an unspecified factor. No theory of such a process has been proposed yet. Also, the moment when the disc reaches the ISCO in transients, and the hot inner flow/outer disc configuration becomes replaced by a disc-corona geometry (at a luminosity much below that of the state transition) has to have no observational signature. This lack of an observational signature, in particular no change in the spectral index, is difficult to understand, given the radical change of the energy balance between the hot and cold components predicted in the two cases (e.g., Poutanen \& Svensson 1996; Poutanen, Krolik \& Ryde 1997; Zdziarski, Lubiński \& Smith 1999; Poutanen \& Vurm 2009).

Finally, studies of the response of the blackbody component to changes in the hot component flux in the hard state of BH binaries show rather long time lags, corresponding to the distance between the disc and the bulk of the coronal emission of at least several tens of the gravitational radii (De Marco et al. 2015, 2017; De Marco \& Ponti 2016). This is again consistent with the truncated disc geometry.

Given those major issues, we have decided to study again the excellent spectral data from simultaneous Suzaku (Mitsuda et al. 2007) and NuSTAR (Harrison et al. 2013) observations of Cyg X-1, investigated before by P15. NUSTAR, with its unprecedented focusing capability in a wide band covering soft to hard X-rays (3$79 \mathrm{keV}$ ), minimal pile-up, low background and high sensitivity provides a very good quality spectrum which is essential for such studies. In addition, Suzaku extends the energy coverage to both lower and higher energies with a good spectral resolution, which is useful to simultaneously model the disc emission, continuum, reflection hump and the cutoff energy. P15 found the reflecting disc extending down close to the ISCO of a BH with the spin near the maximum value, and irradiated by a lamppost very close to the horizon. They assumed the continuum to be either an e-folded power law or a due to emission of a single source containing hybrid (thermal and non-thermal) electrons.

However, there appears to be strong evidence that the 1-10 $\mathrm{keV}$ underlying spectrum is not given by a single component. First, broad-band spectral fits of Cyg X-1 in the hard state of Gierliński et al. (1997), Di Salvo et al. (2001), Frontera et al. (2001), Makishima et al. (2008) and Nowak et al. (2011) required an additional soft component in addition to the disc, hard Comptonization and reflection components. Then, Yamada et al. (2013) have analysed hardstate Cyg X-1 data from Suzaku using temporal spectroscopy finding that at least two Comptonization components, hard and soft, are required. These components can be interpreted as an approximation for an inhomogeneous Comptonization cloud, with the spectra hardening with the decreasing radius. This scenario is also compatible with the X-ray hard lags observed in the hard state of Cyg X-1 (Kotov, Churazov \& Gilfanov 2001), interpreted as propagating fluctuations in the presence of the hardening locally-emitted spectra. Indeed, Revnivtsev, Gilfanov \& Churazov (1999) studied Fourier-resolved spectra of Cyg X-1 in the hard state and found the spectra harden with the increase of the considered range of Fourier frequency. The same result has been obtained using the timescaleresolved spectrscopy (Wu et al. 2009). Furthermore, Wu, Belloni $\&$ Stella (2010) have found that the X-ray spectra of the hard state of Cyg X-1 fluctuate on time scales $\lesssim 1 \mathrm{~s}$, changing their $2-20 \mathrm{keV}$ photon spectral index in the range of at least $\Gamma \simeq 1.6-1.8$, with $\Gamma$ positively correlated with the flux. This also implies that the timeaveraged spectrum is concave, showing a soft excess at low energies, see discussion in Section 6.1. Therefore, in our analysis we allow for the presence of two separate Comptonization components.

Our paper is organized as follows. In Section 2 we review the parameters of Cyg X-1 and describe the method of analysis. Section 3 describe the data reduction and Section 4 presents our treatment of the wind absorption. Section 5 presents our spectral analysis, 
where we first compare directly our results with those of P15, and then develop our own approach. We discuss our results in Section 6 and present the conclusions in Section 7.

\section{THE PARAMETERS OF CYG X-1}

Cyg X-1 is one of the first detected X-ray sources (Bowyer et al. 1965). The current distance determinations are $1.86_{-0.11}^{+0.12} \mathrm{kpc}$ based on radio parallax (Reid et al. 2011)) and $1.81 \pm 0.09 \mathrm{kpc}$ (Xiang et al. 2011) based on a study of dust scattering (though the latter discarded three models yielding larger distances as incompatible with the former). It contains a $\mathrm{BH}$ with the mass estimated as $14.8 \pm 1.0 \mathrm{M}_{\odot}($ Orosz et al. 2011). The value of the BH spin appears uncertain, with some measurements giving the dimensionless spin, $a_{*}$, close to unity (Gou et al. 2011; Fabian et al. 2012; Tomsick et al. 2014), while Kawano et al. (2017) obtain $a=0.80_{-0.30}^{+0.08}$. The companion, the 09.7 Iab supergiant HDE 226868 (Walborn 1973) has a relatively uncertain mass of $17-35 \mathrm{M}_{\odot}$ (Orosz et al. 2011; Ziółkowski 2014). The binary inclination is probably in the range of $\sim 25^{\circ}-35^{\circ}$ (Orosz et al. 2011; Ziółkowski 2014).

The donor emits strong stellar wind, with the mass-loss rate estimated as $\sim 2.5 \times 10^{-6} \mathrm{M}_{\odot} \mathrm{y}^{-1}$ (Hutchings 1976; Gies et al. 2003). At the relatively low separation between the stars of $3 \times 10^{12} \mathrm{~cm}, \mathrm{X}$-ray photons emitted close to the BH suffer substantial attenuation in the wind, which attenuation peaks at the superior conjunction. The observations studied here were done around it, with the range of the orbital phases of the NUSTAR observation of 0.90-0.10, and that for Suzaku, 0.73-0.28. Thus, we need to take particular care of the wind absorption.

The wind of Cyg X-1 consists of a smooth phase and clumps. For the smooth wind phase, we use a model of an ionized absorber based on the XSTAR code version 2.2.1bg (Kallman \& Bautista 2001), which was also used by Tomsick et al. (2014). This is a table model with the elemental abundances assumed to be solar, the constant $\mathrm{H}$ number density of $10^{12} \mathrm{~cm}^{-3}$ and the turbulent velocity of $300 \mathrm{~km} \mathrm{~s}^{-1}$. The free parameters are the ionization parameter, $\xi_{\mathrm{w}}$ (in units of $\mathrm{erg} \mathrm{cm} \mathrm{s}^{-1}$ ) and the column density, $N_{\mathrm{Hw}}$ (in units of $\mathrm{cm}^{-2}$ ), which can be varied in a logarithmic grid in the ranges of $10^{2} \leq \xi_{\mathrm{w}} \leq 10^{5}$ and $1.0 \times 10^{21} \leq N_{\mathrm{Hw}} \leq 5.0 \times 10^{22}$. We then exclude X-ray dips present in the Suzaku soft X-ray light curve due to the clumps (Bałucińska-Church et al. 2000; Poutanen, Zdziarski \& Ibragimov 2008), see Section 4.

The column density of the interstellar medium (ISM) towards Cyg X-1 has been estimated as $N_{\mathrm{H}} \simeq(6 \pm 2) \times 10^{21} \mathrm{~cm}^{-2}$ based on the reddening towards the donor (Balucinska-Church et al. 1995). Tomsick et al. (2014) then fitted the soft state of Cyg X-1 including absorption by the stellar wind. That state has much stronger soft emission, allowing for much better determination of the column than the hard state. They found $N_{\mathrm{H}} \simeq 6.0 \pm 0.3,6.2 \pm 0.2 \times 10^{21}$ $\mathrm{cm}^{-2}$. Allowing for some additional uncertainty, we constrain our fitted $N_{\mathrm{H}}$ to $(6 \pm 1) \times 10^{21} \mathrm{~cm}^{-2}$. We use the model TBnew ${ }^{1}$ with the abundances of Anders \& Grevesse (1989).

\section{THE DATA REDUCTION}

Table 1 summarizes the observations used for our analysis. Suzaku and NUSTAR observed the source 2014 May 19-22 and May 20-

\footnotetext{
1 http://pulsar.sternwarte.uni-erlangen.de/wilms/ research/tbabs
}

21 , respectively. In the data reduction, we follow the standard procedures, similarly to P15, but with the calibration and software updated, as specified below.

The Suzaku data have the observation ID 409049010. The available data from the X-ray Imaging Spectrometer (XIS; Koyama et al. 2007) are from the XIS1 unit only, which was the only one operational at that time due to the limited available power, and for which we extract only the data simultaneous with the NUSTAR. The XIS observation was taken in the $1 / 4$-window mode to cope with the high count rate of Cyg X-1. We use the CALDB files of 2016 June 07 and the HEASOFT v. 6.19. The task aepipeline is used to make the event list, followed by the tasks aeattcor 2 and xiscoord to obtain the updated photon positions. The pileest task shows a significant pile-up within the inner radius of $1^{\prime}$ of the point-spread function (PSF), in spite of using the 1/4-window mode. Hence, we use an annular region with an inner and outer radii of $1^{\prime}$ and $4^{\prime}$, respectively, for the data extraction. The pile-up fraction is now reduces to only 1.6 per cent when averaged over photons, or 0.7 per cent averaged over pixels. The background is extracted from a rectangular region near the edge of the exposed detector with a similar area as that of the source extraction region. As a part of the source extraction region lies on the inactive region of the detector, we adjust the BACKSCAL keyword of the source spectral files. Finally, the tasks xisrmfgen and xissimarfgen were used to create the response and ancillary files. We bin the XIS data with an oversampling factor of 3 with the minimum signalto-noise ratio of 30 . We add in quadrature a 0.5 per cent of the systematic error to the XIS1 spectral data (Tsujimoto et al. 2011).

The Hard X-ray Detector (HXD; Takahashi et al. 2007) of Suzaku consists of Si PIN detectors and GSO scintillators; the data from them were extracted using the PERL scripts hxdpinxbpi and hxdgsoxbpi, respectively. The non-X-ray background for both of the detectors were obtained from the public sites ${ }^{2}$. In addition, the cosmic X-ray background for the PIN was obtained based on the HEAO observations of Gruber et al. (1999). The response files for the PIN and GSO are ae_hxd_pinxinome11_20110601.rsp and ae_hxd_gsoxinom_20100524.rsp, respectively, and a GSO ancillary file, ae_hxd_gsoxinom_crab_20100526.arf, is used. We add 1 per cent systematic error to the spectra from both detectors (Ota et al. 2008; Bautz et al. 2009). We use the entire HXD data set (as in P15), given the very modest spectral variability during the observation.

The NUSTAR data have the observation ID 30001011007. To process them, we used the NuSTARDAS v. 1.4.1 and the CALDB of 2015 March 16. We used NUPIPELINE and NUPRODUCTS routines to extract the cleaned event files and the spectral products, respectively. The source region was chosen as a circle with a radius $150^{\prime \prime}$ centred at the position of Cyg X-1, while the background was chosen as a circle with a radius $100^{\prime \prime}$ away from the source. The data were binned with an oversampling factor of 3 and a minimum of 50 counts per spectral bin. We add 0.5 per cent systematic error to the data (Madsen et al. 2015).

The data and the background of all the detectors are found to be very similar to those obtained by P15. We have used the following energy ranges for the spectral analysis (similar to P15). For XIS, we choose the $1.2-9.0 \mathrm{keV}$ range but excluding the $1.7-2.5 \mathrm{keV}$ range, where there are uncertain calibration issues related to the $\mathrm{Si}$

\footnotetext{
2 ftp://legacy.gsfc.nasa.gov/suzaku/data/background/ pinnxb_ver2.2_tuned and ftp://legacy.gsfc.nasa.gov/suzaku/ data/background/gsonxb_ver2.6
} 
Table 1. The analysed observations of Cyg X-1 with NuSTAR and Suzaku. The used value of the Eddington luminosity, $L_{\mathrm{E}}$, is for the BH mass of $14.8 \mathrm{M} \odot$ and the $\mathrm{H}$ fraction of 0.7 , the source flux is unabsorbed, and the luminosity, $L$, corresponds to this flux at the distance of $1.86 \mathrm{kpc}$ assuming isotropy.

\begin{tabular}{|c|c|c|c|c|c|c|c|}
\hline Detector & Start time (UT) & End time (UT) & $\begin{array}{c}\text { Effective } \\
\text { exposure (ks) }\end{array}$ & $\begin{array}{c}\text { Count rate } \\
\mathrm{s}^{-1}\end{array}$ & $\begin{array}{c}F\left(4-79 \mathrm{keV}^{(a)}\right. \\
10^{-8} \mathrm{erg} \mathrm{cm}^{-2} \mathrm{~s}^{-1}\end{array}$ & $\begin{array}{c}F\left(0.01-10^{3} \mathrm{keV}\right)^{(a)} \\
10^{-8} \mathrm{erg} \mathrm{cm}^{-2} \mathrm{~s}^{-1}\end{array}$ & $L / L_{\mathrm{E}}$ \\
\hline XIS1 & 2014-05-20 06:40:34 & 2014-05-22 07:20:40 & $12.0^{(c)}$ & 123.5 & & & \\
\hline PIN & 2014-05-19 06:48:19 & 2014-05-22 07:27:16 & 107.0 & 9.7 & & & \\
\hline GSO & 2014-05-19 06:48:19 & 2014-05-22 07:27:16 & 107.0 & 11.7 & & & \\
\hline FPMA & 2014-05-20 05:58:41 & 2014-05-21 08:45:01 & 34.4 & 179.8 & 1.83 & 9.80 & 0.022 \\
\hline FPMB & 2014-05-20 05:58:41 & 2014-05-21 08:45:01 & 35.3 & 163.4 & & & \\
\hline
\end{tabular}

(a) The unabsorbed flux calculated for the normalization of the FPMA detector and Model 1.

(b) 2014-05-21 09:05:15 for the XIS observation simultaneous with the NuSTAR one.

(c) The XIS exposure time simultaneous with NuSTAR is $6.4 \mathrm{ks}$, and that after removing the X-ray dips from it is $4.50 \mathrm{ks}$.

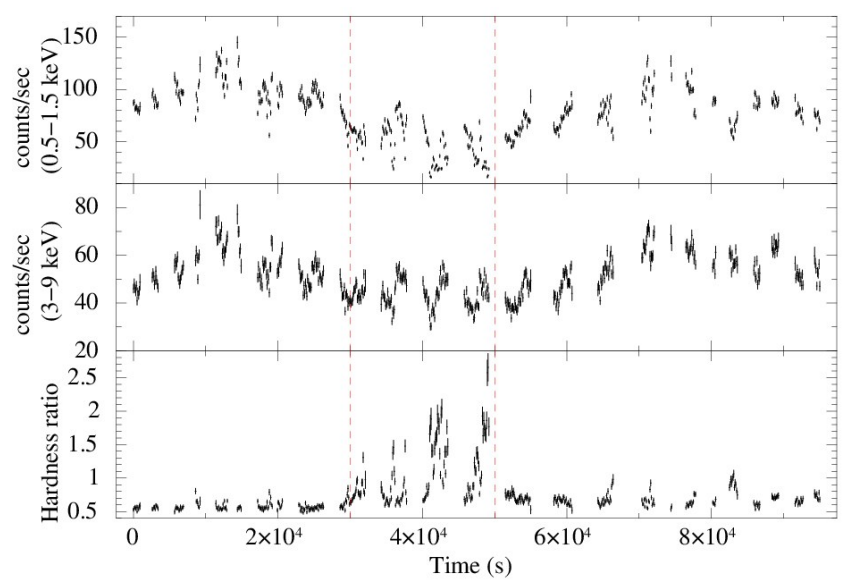

Figure 1. The Suzaku XIS1 light curve in the 0.5-1.5 (top) and $3-9 \mathrm{keV}$ (middle) energy bands during the interval simultaneous with the NuSTAR observation. The bottom panel shows the hardness ratio. The vertical dashed line show the excluded interval containing X-ray dips. Time is measured from the beginning of the XIS1 observation, see Table 1.

K-edge. The HXD PIN data are used in the $20-70 \mathrm{keV}$ range, which excludes the low energy range in order to avoid thermal noise, but also the $38-43 \mathrm{keV}$ range, which has known calibration issues, is excluded. The HXD GSO data are used in the $60-300 \mathrm{keV}$ range. The NuSTAR energy range is chosen as $4-79 \mathrm{keV}$, to mitigate the effect of the X-ray dips, see Section 4. We use XSPEC v. 12.9.0n for the spectral analyses.

\section{X-RAY DIPS}

The presence of clumps causes occasional dips and hardening in soft X-rays. The frequency of dips peaks around the superior conjunction (Bałucińska-Church et al. 2000; Poutanen et al. 2008), and thus we should remove them from the light curve. Those authors used specified spectral hardenings between three energy ranges in the light curve as the criterion for their occurrence. Equivalently, we can use a colour-colour diagram to identify them, following the method of Nowak et al. (2011). Those authors noted that Cyg X-1 has a significant scattering halo, produced by scattering of its light by dust in the ISM (Overbeck 1965; Predehl \& Schmitt 1995; Xiang et al. 2011; Smith, Valencic \& Corrales 2016). This causes an attenuation of the direct flux by scattering of photons away from the line of sight, with the cross section $\propto E^{-3}$. In the case of Cyg

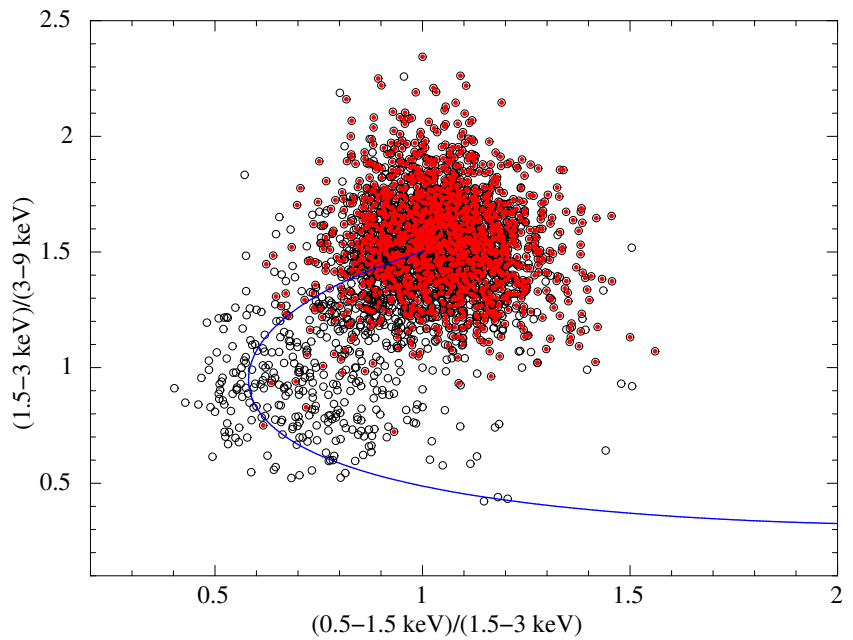

Figure 2. The colour-colour diagram for the XIS1 based on the count rates in the $0.5-1.5,1.5-3$ and 3-9 keV energy bands for the observation simultaneous with NUSTAR (see Fig. 1) for time bin size of $16 \mathrm{~s}$. The black open circles and red filled ones correspond to the observations during the identified dipping interval (within the dashed lines in Fig. 1), and outside it, respectively. The latter is used for the spectral fitting. The solid curve shows the positions of points assuming the $\mathrm{H}$ column density of a wind clump changes from 0 (at the top end of the curve) to $2 \times 10^{23} \mathrm{~cm}^{-2}$ (at the bottom end).

$\mathrm{X}-1$, halo scattering is important at $E \lesssim 3 \mathrm{keV}$. For the point-spread function of either XIS or NUSTAR, this attenuation is compensated by photons emitted to other directions by the source and scattered toward the observer. The light travel time of indirect photons is increased by intervals depending on the scattering position; thus the energy-dependent light curves of halo photons correspond to an average of the past source activity. Therefore, the presence of halo dilutes the X-ray dips. Photons going directly through a wind clump to the observer are attenuated in it, but those coming at the same time from the halo are not. Nowak et al. (2011) take this effect into account by making simplifying assumptions that the delayaveraged spectrum of Cyg X-1 is identical to an instantaneous one and the absorption in the clumps is neutral, see their equation (2).

In Fig. 1, we show the XIS1 light curves and the hardness ratio for the interval simultaneous with the NUSTAR observation. We can clearly see dips occurring, e.g., during the time interval of $3-5 \times 10^{4} \mathrm{~s}$, when the hardness ratio strongly increases. We exclude this interval, as shown by the dashed vertical lines in Fig. 1. Fig. 2 shows the colour-colour diagram for these data obtained using the 


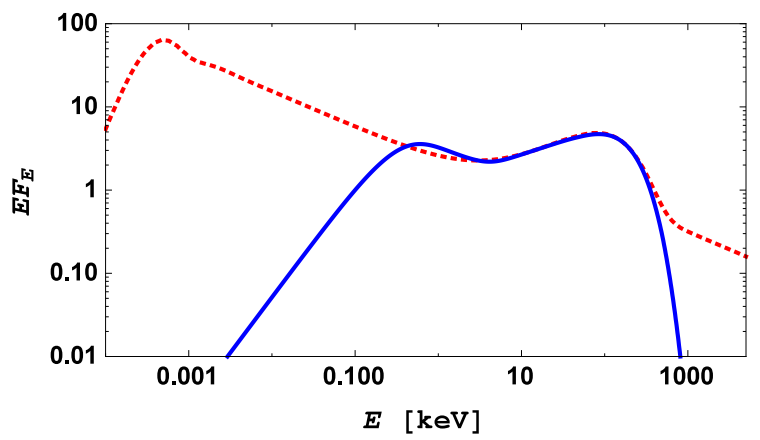

Figure 3. The red dotted curve shows the hybrid Comptonization spectrum obtained as the best-fit continuum to the Cyg X-1 spectrum by P15. Blackbody photons at $k T=0.11 \mathrm{eV}$ are Comptonized by a hot plasma, which thermal part has the electron temperature of $70 \mathrm{keV}$ and the Thomson optical depth of 0.57 . This yields a power-law spectrum from about $3 \mathrm{eV}$ to 500 $\mathrm{eV}$ with the photon index of $\Gamma \simeq 2.4$. The hump beyond that is due to thermal bremsstrahlung, and it is visible because of the very low compactness parameter, equal to $\simeq 0.03$ for the total spectrum (and several times less for photons at $E>1 \mathrm{keV}$ ). Beyond the bremsstrahlung hump, there is a tail due to both bremsstrahlung and single Compton upscattering by non-thermal electrons, at the fitted ratio of the non-thermal to thermal luminosities of $\sim 0.1$. The blue solid curve shows our double thermal Comptonization spectrum for Model 1, also shown with its additional components in Fig. 4(b); see Table 2 for the parameters.

method of Nowak et al. (2011). We show the ratio of the XIS1 count rates in the energy bands of $0.5-1.5 \mathrm{keV}, 1.5-3 \mathrm{keV}$ and $3-9 \mathrm{keV}$ during the interval with dips and outside it. The solid curve in Fig. 2 shows the trajectory expected for dips caused by wind clumps with the $\mathrm{H}$ column density between 0 and $2 \times 10^{23} \mathrm{~cm}^{-2}$ obtained using equation (2) of Nowak et al. (2011), and assuming the continuum is represented by disc blackbody and a power law absorbed by a neutral medium. We can see that it only approximately determines the part of the diagram affected by the dips, with some dipping events less absorbed in the $0.5-1.5 \mathrm{keV}$ range, which is due to our simplifying assumption of the absorption by a wind clump being neutral.

We exclude the dips only for the XIS1 data, which then reduces the effective exposure of the part simultaneous with $N u S$ $T A R$ to $4.5 \mathrm{ks}$. Given that NUSTAR has the light curve much more densely sampled than that by XIS, we cannot remove dipping from the NUSTAR light curve based on XIS. Dipping does affect the 3-4 $\mathrm{keV}$ energy range of NUSTAR, but given that we have no data below $3 \mathrm{keV}$ for that instrument, we cannot exclude dips in those light curves based on those data. Therefore, we do not take into account the NUSTAR 3-4 keV energy range in our fitting.

\section{SPECTRAL ANALYSIS}

\subsection{Comparison with Parker et al. (2015)}

P15 have analyzed the Suzaku/NuSTAR spectra in three different ways. First, they considered the broad-band spectrum fitted in a simple way by Galactic absorption, disc blackbody and thermal Comptonization, with the intention to visualise systematic departures from that model. Here, we obtain similar results.

Second, P15 considered the $4-10 \mathrm{keV}$ range from both XIS and NuSTAR, for which they used the full XIS spectrum and used the relxilllp model only. They found the disc extending almost to the ISCO, $r_{\text {in }}=1.11 \pm 0.01 r_{\text {ISCO }}$, where both the inner and the
ISCO radii are in units of the gravitational radius, $R_{\mathrm{g}}=G M / \mathrm{c}^{2}$. Here, we use only the spectra simultaneous with NUSTAR. Our results are consistent with those of P15.

Third, P15 considered physical broad-band continua. They found no good fit with a single thermal Comptonization continuum, modelled by comptt (Titarchuk 1994), together with disc blackbody and reflection. We obtain the same result, using the nthComp Comptonization model (Zdziarski, Johnson \& Magdziarz 1996). We find $\Delta \chi^{2} \simeq+700$ with respect to our best-fit with two Comptonization continua (Model 1), see Section 5.2. This clearly rules out a single Comptonization and a disc blackbody as the underlying continuum in $\mathrm{Cyg} \mathrm{X}-1$, in agreement with a number of previous papers, see Section 1.

On the other hand, P15 found an excellent fit using the eqpair ${ }^{3}$ model (Poutanen \& Coppi 1998; Coppi 1999; Gierliński et al. 1999). We have refitted that model to the present data and obtained almost identical results to those of P15, namely $a_{*} \simeq 0.98$, $h \simeq 1.1^{+0.8} r_{\mathrm{H}} \simeq 1.3^{+1.0}$ (with 1.1 $r_{\mathrm{H}}$ being the lowest height at which the lamppost model relxilllp is defined), $r_{\text {in }} \simeq(1.6 \pm 0.1) r_{\text {ISCO }} \simeq$ $2.5 \pm 0.2$, and $\chi^{2} \simeq 1232 / 1000$ d.o.f., where $h$ is the height of the lamppost and $r_{\mathrm{H}}=\left(1+\sqrt{1-a_{*}^{2}}\right)$ is the horizon radius ${ }^{4}$ in units of $R_{\mathrm{g}}$. The inclination is $i \simeq 48^{\circ} \pm 1^{\circ}$, and the relative Fe abundance is $A_{\mathrm{Fe}} \simeq 4.1_{-0.2}^{+0.3}$. The best fit ISM column density is $4.2 \times 10^{21} \mathrm{~cm}^{-2}$, which is outside our adopted range, see Section 2. Constraining it to $\geq 5 \times 10^{21} \mathrm{~cm}^{-2}$ leads to $\Delta \chi^{2} \simeq+54$.

Unfortunately, there are some objections to the physical nature of the adopted incident continuum, which spectrum we show in Fig. 3. First, the unit of the seed blackbody temperature in eqpair is $\mathrm{eV}$, but it was tied to the diskbb temperature, which is in $\mathrm{keV}$. Thus, the disc blackbody component had the temperature of $0.11 \mathrm{eV}$. Second, and more importantly, the total compactness, $\ell \equiv L \sigma_{\mathrm{T}} / D m_{\mathrm{e}} c^{3}$, where $L$ and $D$ are the luminosity and source size, was fitted as $\sim 2.8 \times 10^{-2}$. This compactness implies the X-ray source size as large as $R \sim 3 \times 10^{11} \mathrm{~cm}$. For comparison, $D=0.1 R_{\mathrm{g}}$ at the observed luminosity implies $\ell \simeq 5000$, a compactness five orders of magnitude higher than that fitted in P15. Furthermore, most of the model flux was below $1 \mathrm{keV}$, see Fig. 3, while that of Cyg X-1 is a small fraction of the bolometric one, which adds one more order of magnitude to the discrepancy. Also, the hard-to-soft compactness ratio was fitted as $\simeq 1.2$. These parameters imply that the slope of the thermal Comptonization is $\Gamma \simeq 2.4$, much softer than $\Gamma \simeq 1.6$ found as the overall broad-band slope by P15. This slope is seen at low energies in Fig. 3. The hard component in the data is then accounted for by thermal bremsstrahlung, which becomes dominant above $\sim 2 \mathrm{keV}$, and which is visible only because of the very weak Comptonization cooling at the fitted very low compactness. Obviously, this model cannot correspond to the real physical situation in $\mathrm{Cyg} X-1^{5}$. Still, its good fit to the overall spectrum reiterates

\footnotetext{
3 http://www .astro.yale.edu/coppi/eqpair/eqpap4.ps

4 We note that while P15 stated their values of $h$ are in units of $R_{\mathrm{ISCO}}$, they were in units of the horizon radius.

5 In fact, as found by Ibragimov et al. (2005), no fit with a single hybrid Comptonization model for Cyg X-1 can be obtained using realistic constraints on the source size. This was confirmed by P15, who has searched the entire parameter space and found no solution except for the one dominated by bremsstrahlung in hard X-rays. The reason for that is that Comptonization by the thermal electrons always dominates at low energies, while that by non-thermal ones can dominate only at the highest energies, and thus cannot reproduce the observed cutoff at $\sim 100 \mathrm{keV}$ (while it can be responsible for the MeV-range tail, e.g., McConnell et al. 2002; Poutanen \& Vurm 2009).
} 
the importance of taking into account the presence of the soft excess in Cyg X-1 (as also discussed in Section 1). Fig. 4(a) shows this model with its additional components, disc blackbody and reflection, and Fig. 5(a) shows the fit residuals. We consider below physical models that yield similar underlying continua.

\subsection{Models with two Comptonization regions}

We consider here two thermal Comptonization components, hard and soft, each modelled with nthComp. We add relativistically blurred reflection to either only to the dominant hard component (Model 1) or to both (Model 2). We model reflection with either relxillcp or relxilllpcp (García \& Kallman 2010; Dauser et al. 2010; García et al. 2014), which models use the above thermal-Compton model for the incident continuum. We note that they assume that the maximum temperature of disc blackbody photons (which serve as seeds for Comptonization) is $0.1 \mathrm{keV}$, without allowing it to vary (J. García, private communication). Therefore, we use them for reflection only, and still use nthComp for Comptonization spectra. At low energies, we include blackbody emission from the disc, which we model using diskbb (Mitsuda et al. 1984; Makishima et al. 1986). The input seed photons for each of the nthComp Comptonization components are assumed to have a disc blackbody distribution with the same temperature as the diskbb component. However, the spectrum at energies $<1 \mathrm{keV}$ appears more complex than a simple disc blackbody (see, e.g., results of Basak \& Zdziarski 2016), which study is not the focus of this work. Thus, we consider the data at energies above $1.2 \mathrm{keV}$ only, similarly to P15. In relxillCp and relxilllpCp, we assume the maximum value of the spin parameter, $a_{*}=0.998$ (cf. Section 2), at which value $r_{\mathrm{ISCO}} \simeq 1.24$ and $r_{\mathrm{H}} \simeq 1.13$. However, given that we find $r_{\text {in }}$ substantially above the ISCO in most models, this value has a little effect on our results. The outer radius of the accretion disc is set to $1000 R_{\mathrm{g}}$ and the radial emissivity power-law index is set to 3 , though in one model we fit it.

We first consider the case with no reflection of the soft Compton component (Model 1). The fitting results are shown in Table 2. The $\chi^{2} \simeq 1249 / 1004$ d.o.f. is slightly worse than that of the eqpair model, see Section 5.1. The model yields $r_{\text {in }} \simeq 6 \pm 2$, which corresponds to a truncated disc at the assumed maximum spin, but it can reach ISCO for lower spin values. We also find $i \simeq 25_{-4}^{+5 \circ}$, compatible with the modelling of the binary, see Section 2. The soft-Compton component has $\Gamma \simeq 2.6$ and a very low temperature of $k T_{\mathrm{e}} \simeq 0.7 \mathrm{keV}$. Fig. 4(b) shows the unabsorbed model components, and Fig. 5(b) shows the residuals. In the former figure, we see the strong contribution of the soft-Compton component at low energies, exceeding that of the hard Comptonization below $\simeq 1.5$ $\mathrm{keV}$.

We then add reflection to the soft Compton component (Model 2). This significantly improves the fit, to $\chi^{2} \simeq 1226 / 1003$, i.e., substantially better than the eqpair model of P15, which has the $\chi^{2}$ higher by 6 in spite of three more free parameters. The fitting results are shown in Table 2. Fig. 4(c) shows the unabsorbed model components, and Fig. 5(c) shows the residuals, The model yields $r_{\text {in }} \simeq 17 \pm 3$, i.e., a significantly truncated disc. The soft-Compton component has $\Gamma \simeq 2.6$ and a low temperature of $k T_{\mathrm{e}} \simeq 5.0 \mathrm{keV}$. The main differences with respect to the previous model are that now the soft component is reflection-dominated and both components contribute to the $\mathrm{Fe} \mathrm{K} \alpha$ line. The dominance of reflection, at face value, requires shielding of a part of the disc. However, it may indicate the presence of a spectral component additional to the two Compton ones of Model 1, and/or some spectral complexity
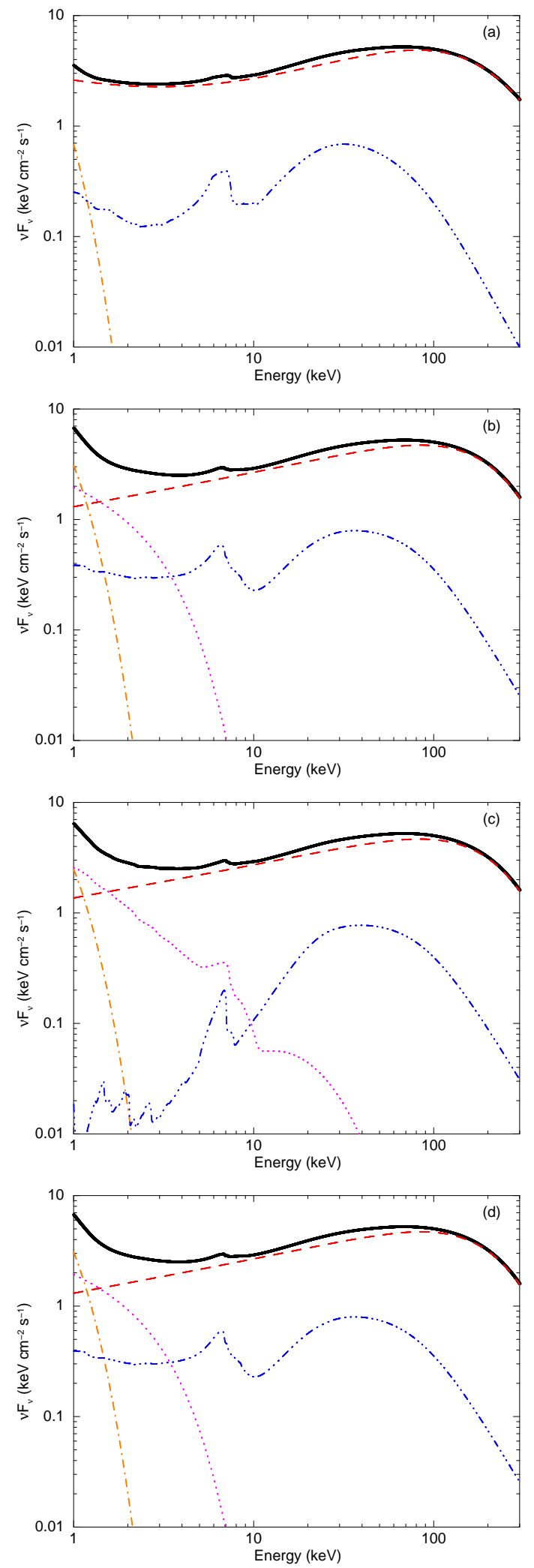

Figure 4. The unabsorbed $E F_{E}$ spectra of (a) the model of P15; (b) Model 1, (c) Model 2, and (d) Model 4. The (red) dashes show the primary incident continuum, the (magenta) dotted curves show the additional soft continua (the panels $\mathrm{b}$ and $\mathrm{c}$ only), the (orange) dot-dashed curves show the disc blackbody, and the (blue) triple-dot-dashed curves show the reflection of the primary continua. The (black) solid curves show the sum. 
Table 2. The results of spectral fitting for our main models. All the models have absorption by the ISM and the wind of tbnew*xstar. Their continua are: Model 1: diskbb+nthComp+relxillCp+nthComp; Model 2: diskbb+nthComp+relxillCp+relxillCp; Model 3: diskbb+nthComp+relxillCp+relxillCp+xillverCp; Model 4: diskbb+nthComp+relxilllpCp+nthComp.

\begin{tabular}{|c|c|c|c|c|c|}
\hline Component & Parameter & Model 1 & Model 2 & Model 3 & Model 4 \\
\hline ISM absorption & $n_{\mathrm{H}} / 10^{22}$ & $0.70_{-0.07}^{+0}$ & $0.70_{-0.10}^{+0}$ & $0.70_{-0.08}^{+0}$ & $0.70_{-0.07}^{+0}$ \\
\hline Wind absorption & $\begin{array}{c}n_{\mathrm{Hw}} / 10^{22} \\
\log _{10} \xi_{\mathrm{w}}\end{array}$ & $\begin{array}{l}1.78_{-0.37}^{+0.19} \\
4.34_{-0.05}^{+0.16} \\
\end{array}$ & $\begin{array}{l}2.56_{-0.19}^{+0.17} \\
5.00_{-0.08}^{+0}\end{array}$ & $\begin{array}{l}2.49_{-0.21}^{+0.18} \\
5.00_{-0.44}^{+0}\end{array}$ & $\begin{array}{l}1.77_{-0.35}^{+0.18} \\
4.33_{-0.04}^{+0.16}\end{array}$ \\
\hline Disc blackbody & $\begin{array}{c}k T_{\mathrm{bb}} \\
N / 10^{6}\end{array}$ & $\begin{array}{c}0.14_{-0.01}^{+0.04} \\
7.0_{-4.4}^{+2.8}\end{array}$ & $\begin{array}{c}0.14_{-0.01}^{+0.02} \\
5.1_{-3.2}^{+7.5}\end{array}$ & $\begin{array}{c}0.15 \pm 0.01 \\
3.8_{-2.7}^{+4.8}\end{array}$ & $\begin{array}{c}0.14_{-0.02}^{+0.04} \\
6.8_{-3.7}^{+24}\end{array}$ \\
\hline $\begin{array}{l}\text { Hard Comptonization } \\
\text { and reflection }\end{array}$ & $\begin{array}{c}\Gamma \\
k T_{\mathrm{e}} \\
N \\
A_{\mathrm{Fe}} \\
\log _{10} \xi \\
\mathcal{R} \\
i \\
r_{\text {in }} \\
h\end{array}$ & $\begin{array}{c}1.70 \pm 0.01 \\
93_{-6}^{+7} \\
1.30_{-0.03}^{+0.02} \\
4.4_{-0.3}^{+0.4} \\
3.28_{-0.07}^{+0.05} \\
0.19 \\
25_{-4}^{+5} \\
6.3_{-1.8}^{+2.2} \\
-\end{array}$ & $\begin{array}{c}1.71 \pm 0.01 \\
98 \pm 7 \\
1.36 \pm 0.03 \\
2.4_{-0.4}^{+0.3} \\
2.00_{-0.16}^{+0.15} \\
0.18 \\
42_{-2}^{+3} \\
17.1_{-2.6}^{+3.5} \\
-\end{array}$ & $\begin{array}{c}1.71 \pm 0.01 \\
98_{-6}^{+9} \\
1.36 \pm 0.03 \\
2.2 \pm 0.2 \\
2.00_{-0.13}^{+0.22} \\
0.16 \\
43_{-2}^{+3} \\
15.7_{-2.6}^{+3.2} \\
-\end{array}$ & $\begin{array}{c}1.70_{-0.002}^{+0.01} \\
94 \pm 6 \\
1.31_{-0.02}^{+0.01} \\
4.6 \pm 0.2 \\
3.28_{-0.07}^{+0.04} \\
0.20 \\
24 \pm 3 \\
1.24_{-0}^{+6.10} \\
9.3_{-0.5}^{+1.8}\end{array}$ \\
\hline $\begin{array}{l}\text { Soft Comptonization } \\
\text { and reflection }\end{array}$ & $\begin{array}{c}\Gamma \\
k T_{\mathrm{e}} \\
\log _{10} \xi \\
N / 10^{-2}\end{array}$ & $\begin{array}{c}2.61_{-0.65}^{+0.33} \\
0.67_{-0.06}^{+0.15} \\
- \\
195_{-99}^{+48} \\
\end{array}$ & $\begin{array}{c}2.66 \pm 0.10 \\
4.90_{-0.30}^{+1.10} \\
4.00_{-0.14}^{+0.18} \\
2.6_{-0.6}^{+0.3}\end{array}$ & $\begin{array}{c}2.65 \pm 0.08 \\
5.06_{-0.75}^{+1.01} \\
4.00_{-0.10}^{+0.10} \\
2.6 \pm 0.3\end{array}$ & $\begin{array}{c}2.60_{-0.46}^{+0.23} \\
0.66_{-0.06}^{+0.07} \\
- \\
192_{-39}^{+41}\end{array}$ \\
\hline Static reflection & $\log _{10} \xi$ & - & - & $\begin{array}{c}0.68_{-0.68}^{+2.07} \\
0.02\end{array}$ & $\begin{array}{l}- \\
-\end{array}$ \\
\hline $\begin{array}{l}\text { Detector normalization } \\
\text { with respect to FPMA }\end{array}$ & $\begin{array}{c}\text { XIS } \\
\text { PIN } \\
\text { GSO } \\
\text { FPMB }\end{array}$ & $\begin{array}{l}0.90 \\
1.28 \\
1.16 \\
1.02\end{array}$ & & & \\
\hline & $\chi^{2} /$ d.o.f. & $1249 / 1004$ & $1226 / 1003$ & $1216 / 1001$ & $1246 / 1003$ \\
\hline
\end{tabular}

Notes: $n_{\mathrm{H}}$ and $n_{\mathrm{Hw}}$ are the $\mathrm{H}$ column densities (in the unit of $\mathrm{cm}^{-2}$ ) of the ISM and the stellar wind, respectively, with the former constrained to the $(5-7) \times 10^{21} . \xi_{\mathrm{w}}$ and $\xi$ are the ionization parameters (in the unit of $\mathrm{erg} \mathrm{cm} \mathrm{s} \mathrm{cm}^{-1}$ ) of the wind and the reflector, respectively, with the former constrained to $\leq 10^{5} . k T_{\mathrm{bb}}$ and $k T_{\mathrm{e}}$ are the maximum disc and electron temperature (in keV), respectively, $\Gamma$ is the Comptonization photon power-law index, $A_{\mathrm{Fe}}$ is the reflector $\mathrm{Fe}$ abundance in the solar unit, $i$ is the reflector inclination in degrees, and $r_{\text {in }}$ and $h$ are the inner disc radius and the lamppost height, respectively, in

units of $R_{\mathrm{g}}$, and $N$ is a component normalization. Then $\mathcal{R}$ is the strength of the hard reflection defined as the ratio of the $20-40 \mathrm{keV}$ flux of the reflected component to the incident one. Models 1 and 4 have no soft reflection, while it is dominant in Models 2 and 3, i.e., the direct flux is negligible

Correspondingly, the normalization is given as either that of nthComp or relxillCp. The uncertainties are given for 90 per confidence for one parameter of interest, i.e., $\Delta \chi^{2}=2.71$. The relative detector normalizations are the same for all of the shown models.

still not reproduced by the current models. The fitted inclination is found to be $i \simeq 42_{-2}^{+3 \circ}$. We note that the best-fit model of P15 yields even higher inclination, $i \simeq 48^{\circ} \pm 1^{\circ}$, while the soft-state studies of Tomsick et al. (2014) and Walton et al. (2016) yield $i \sim 40^{\circ}$. All these values are significantly larger than the binary inclination of Orosz et al. (2011) of $27 \cdot 1^{\circ} \pm 0.8^{\circ}$. On the other hand, the analysis of Ziółkowski (2014) points to higher values, $\simeq 30^{\circ}$ or more. Furthermore, it is possible that the inner disc is aligned with the $\mathrm{BH}$ spin axis, which in turn may be different from the binary axis. This appears to be the case in the BH binary GRO J1655-40, which has an accurate determination of the orbital axis inclination of $68.7^{\circ} \pm 1.5^{\circ}$ (Beer \& Podsiadlowski 2002), significantly different from the jet inclination of $85^{\circ} \pm 2^{\circ}$ (Hjellming \& Rupen 1995), with the latter likely to correspond to the BH spin axis. Naturally, systematic inaccuracies of the used model will also contribute to this difference, which, overall, appears to be relatively mild.

We have then searched for spectral solutions with a third thermal Comptonization component, which can be an approximation to the case with a continuous distribution of the temperature and the optical depth of the hot plasma. However, we have not found any model with a significant improvement with respect to the doubleCompton case. Also, we have studied models with the reflection emissivity index different from the standard value of 3 (which we also adopt here), but we found its best fit is $q \simeq 3.00_{-0.36}^{+0.34}$ for our Model 2. Thus, we have kept $q=3$. Given the relatively large disc truncation values for this model, we do not consider a case with the emissivity index increasing close to the $\mathrm{BH}$.

We then consider the possibility of another reflection component from an outer region of a flared disc. The standard accretion disc (Shakura \& Sunyaev 1973) is flared, with the fractional scale height increasing with the radius, $H / R \propto R^{1 / 8}$, which effect becomes even stronger in the presence of irradiation. Such an effect is not included in standard reflection codes, such as relxillcp, which assume a flat reflector. Since relativistic kinematic effects are negligible for outer disc, we use for it the static reflection model xillverCp (García \& Kallman 2010). We add it with the parameters tied to those of the hard Comptonization and blurred reflection components in Model 2, which we denote Model 3. We show the fitting results in Table 2 and the unfolded spectrum and the residuals in Fig. 6. We obtain $\chi^{2} \simeq 1216 / 1001$, which improvement of 

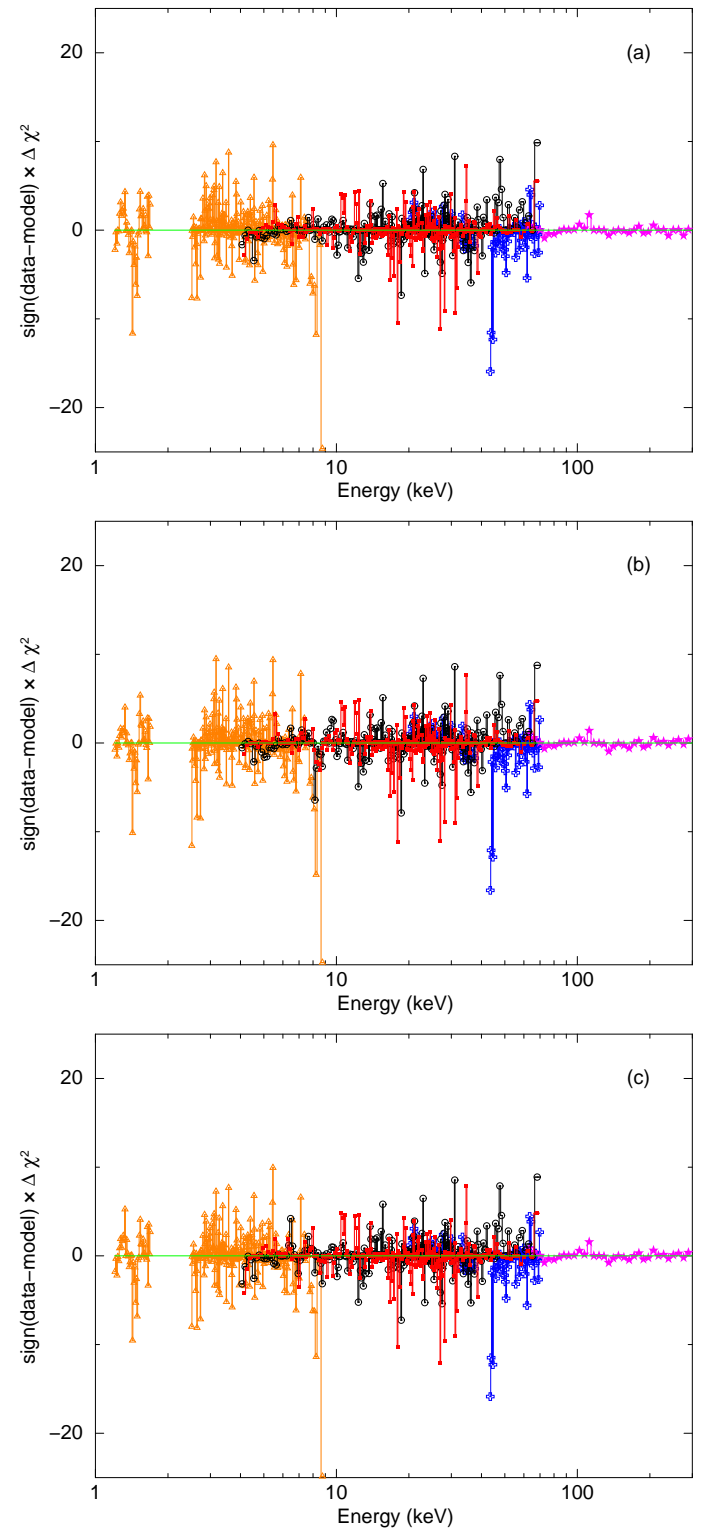

Figure 5. The fit residuals given as $\chi^{2}$ contributions in (a) the model of P15; (b) Model 1 and (c) Model 2. The colours correspond to different detectors: orange: XIS (1.2-9.0 keV, neglecting 1.7-2.5 keV), blue: PIN (20$70 \mathrm{keV}$, neglecting $38-43 \mathrm{keV})$, magenta: GSO $(60-300 \mathrm{keV})$, cyan and red: the NUSTAR FPMA and FPMB, respectively (4-79 keV).

$\Delta \chi^{2} \simeq-10$ has the chance probability of 0.018 according to the F-test. This our overall best model. The reflection strength of the static component is an order of magnitude lower than that of the relativistic reflection. We conclude that the addition of the static reflection is statistically required. We see in Table 2 that its inclusion only slightly changes the other model parameters, in particular $r_{\text {in }} \simeq 16 \pm 3$.

We finally consider a variant of the Model 1 (the model with a soft Comptonization component without its reflection) with a lamppost, using relxilllpCp (Model 4). As in P15, we allow a free normalization of the reflection component. In contrast to the above models, the fit yields a reflecting disc which can extend down to the ISCO, $r_{\text {in }} \simeq 1^{+4.9} r_{\text {ISCO }}$, even closer to the $\mathrm{BH}$ than the original model of P15. However, the height of the lamppost is large $h \simeq 9.3_{-0.5}^{+1.8}$, much above the range obtained by P15. Statistically,

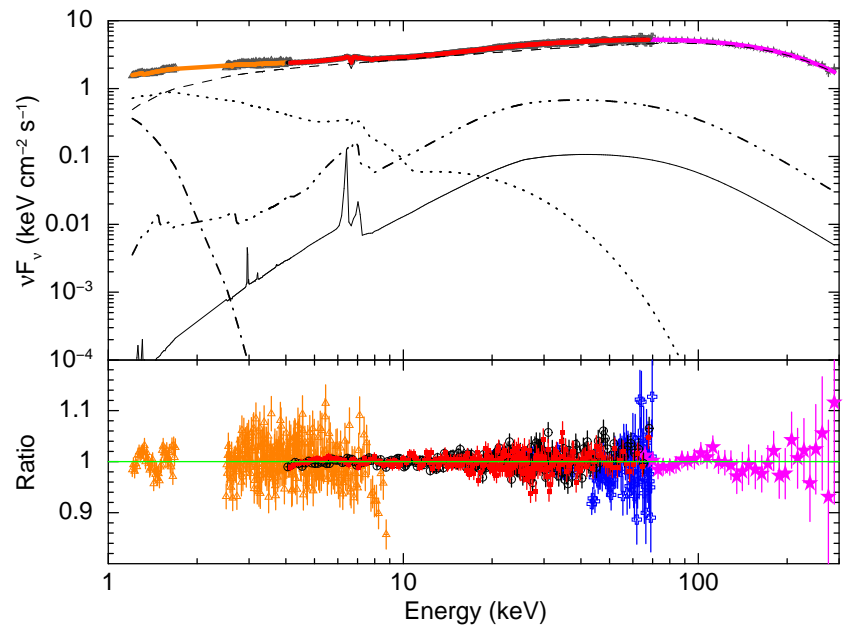

Figure 6. The upper panel shows the unfolded best-fit absorbed $E F_{E}$ data and model spectrum for our overall best Model 3. The model and data are normalized to those of the FMPA detector of NUSTAR. Colours in the data indicate the detectors and are the same as in Fig. 5. The total model is shown by the thick solid curve, while the dot-dashes, dashes, dots, tripledot-dashes and thin solid curve correspond to the disc blackbody, the hard Comptonization, the relativistically blurred reflection of the soft Comptonization and of the hard Comptonization, and the static reflection, respectively, all in black. The lower panel shows the fit residuals given as the data-to-model ratio.

the fit is comparable to our Model 1, with $\chi^{2} \simeq 1246 / 1003$ d.o.f. The fitted inclination is low, and compatible with that of Orosz et al. (2011), but the Fe abundance is high, similar to Model 1. We show the unabsorbed continuum of this model in Fig. 4(d), where we see that the components of this model are indistinguishable from those of Model 1, Fig. 4(b). We discuss the self-consistency of this spectral solution in Section 6.2.

The photon power-law index of the hard Comptonization in all models is $\simeq 1.7$, and the electron temperature is $\sim 100 \mathrm{keV}$, which are typical values for the hard state of both Cyg X-1 and BH binaries in general, see, e.g., Zdziarski \& Gierliński (2004). The inner disc temperature is found in to be $\sim 0.15 \mathrm{keV}$, similar to that found in other studies of Cyg X-1, e.g., Di Salvo et al. (2001). However, the normalization of the diskbb component is found to be high and only poorly constrained. The reason for the lack of significant constraints is that the energy range used for the analysis is $\geq 1.2 \mathrm{keV}$, which does not allow us to constrain it. Furthermore, strong absorption by both the ISM and the stellar wind makes measurements of the unabsorbed soft X-ray spectrum difficult. Thus, the obtained value of the normalization should be treated with caution.

The reflection strength, defined as the ratio of the $20-40 \mathrm{keV}$ flux in the reflection continuum to that in the incident continuum, of the hard-Compton component is found to be $\simeq 0.2$, typical to the hard state (e.g., Gierliński et al. 1997). The relative Fe abundance of the reflector is $A_{\mathrm{Fe}} \simeq 2.0-2.7$ for Models 2 and 3, which values are are much lower than that obtained for the model of P15, $4.1_{-0.2}^{+0.3}$. We consider the lower value to be more probable. On the other hand, Models 1 and 4 still yield similarly high relative Fe abundance of $\simeq$ 4.1-4.8. The ionization of the reflector is much stronger in Models 1 and 4, and thus it significantly contributes to the line broadening, see a discussion in Basak \& Zdziarski (2016). The stellar wind is found to be strongly ionized, $\log _{10} \xi_{\mathrm{w}} \sim 5$. Similar values were found with the same model by Tomsick et al. (2014) and P15. 


\section{DISCUSSION}

\subsection{The soft excess}

Our work has confirmed the necessity of including a soft excess in models of the X-ray spectra of Cyg X-1 in the hard state. We can obtain a good fit to the data only with it, and we have checked that the broad-band best-fit model of P15 also includes an underlying continuum with a strong soft excess. Both analyses could not find even a rough fit without it. A likely origin of the soft excess is an inhomogeneity of the Comptonizing medium, as discussed in Section 1, with the local spectrum becomes harder (and less variable) with the decreasing distance to the $\mathrm{BH}$.

In Section 1, we mentioned the result of Wu et al. (2010) that the $2-20 \mathrm{keV}$ photon spectral index of Cyg X-1 in the hard state is fluctuating and positively correlated with the flux. This implies that the power-law spectrum is pivoting, with the pivot energy being at $E_{\mathrm{p}} \gtrsim 20 \mathrm{keV}$. The average spectrum is then concave, showing a soft excess at low energies, and given by (Zdziarski et al. 2003),

$\langle F(E)\rangle \propto E^{1-\langle\Gamma\rangle} \times \begin{cases}\sinh x / x, & \text { uniform; } \\ \exp \left(x^{2} / 2\right), & \text { Gaussian, }\end{cases}$

where $\langle\Gamma\rangle$ is the average photon index, $x \equiv \Delta_{\Gamma} \ln \left(E / E_{\mathrm{p}}\right)$, and the distribution of $\Gamma$ is flat from $\langle\Gamma\rangle-\Delta_{\Gamma}$ to $\langle\Gamma\rangle+\Delta_{\Gamma}$ in the uniform case, and $\Delta_{\Gamma}$ is the standard deviation in the Gaussian case.

In Fig. 7, we show as a red dotted curve an example of the spectrum of equation (1), for the Gaussian case with $\langle\Gamma\rangle=1.55$, $E_{\mathrm{p}}=20 \mathrm{keV}$, and $\Delta_{\Gamma}=0.55$. We see this spectrum is similar to the spectrum of our Model 1. Thus, such variability can reproduce well the shape of the X-ray spectrum found in our analysis and modelled by two Comptonization components. In this interpretation, the soft excess is due to the softest power-law components in a pivoting, time-dependent, spectrum, while the hard power law is due to the hardest power-law components. Thus, pivoting provides an alternative interpretation to the observed concave spectrum, in which the two Compton components correspond to the extremes of the timedependent spectrum. In the actual situation in the X-ray source, we can have both spatial inhomogeneity and time dependence, either contributing to the observed curvature in the time-averaged spectra.

The normalized flux variance for the pivoting variability is (Zdziarski et al. 2003),

$\frac{\sigma^{2}}{\langle F(E)\rangle^{2}}= \begin{cases}x \operatorname{coth} x, & \text { uniform; } \\ \exp x^{2}-1, & \text { Gaussian. }\end{cases}$

This qualitatively agrees with the rms in the hard state decreasing in the X-ray range, e.g., Gierliński \& Zdziarski (2005), Gierliński et al. (2011). Zdziarski et al. (2003) also note that this type of variability can result in time lags between light curves measured at different energies, which effect has then been calculated by Körding \& Falcke (2004).

\subsection{The lamppost interpretation}

The extreme closeness of the lamppost to the horizon fitted by P15 is unphysical, as we mentioned in Section 1. Those problems are discussed in detail in Niedźwiecki et al. (2016). One is a small fraction of photons emitted by a lamppost very close to the horizon reaching the observer, see fig. 5 in Dovciak \& Done (2015). This requires an increase of the accretion rate, to compensate for that. The increase is so high that the hard-state accretion rate becomes lower than that in the soft state, which is ruled out for Cyg X-1. In the case of the best fit of this model, with $r_{\text {in }}=1.1 r_{\mathrm{H}}, 10$ times more photons

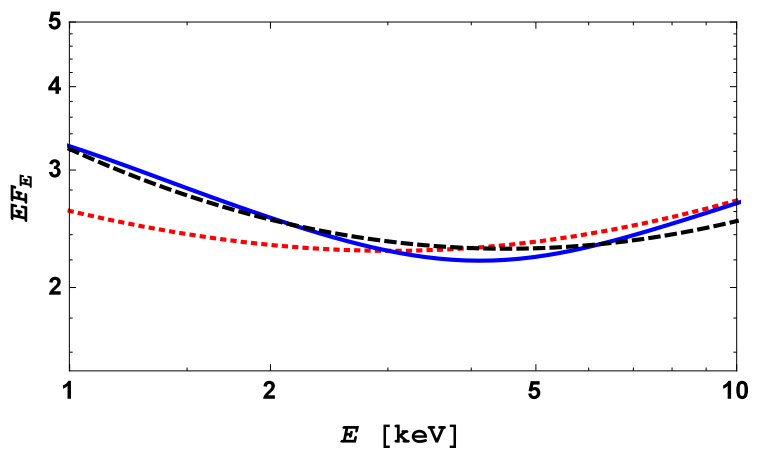

Figure 7. Similar to Fig. 3, but zoomed to the $1-10 \mathrm{keV}$ range. The red dotted curve shows the hybrid Comptonization spectrum of P15, while the blue solid curve shows the incident spectrum of our Model 1. The black dashed curve shows the variable power-law model of equation (1), see Section 6 for the parameters.

falls into the $\mathrm{BH}$ than reaches the observer. The related problem is that the luminosity measured in the local frame is even higher, due to the photon shift and time dilation effects, and the photon energies in the local frame are strongly blueshifted with respect to the measured energies, $1+z=\sqrt{\left(h^{2}+a_{*}^{2}\right) /\left(h^{2}+a_{*}^{2}-2 h\right)}$, where $z$ is the redshift measured at infinity. In the best-fit model of P15, we have $1+z \simeq 7.6$. This causes the source to be strongly out of equilibrium between production of $\mathrm{e}^{ \pm}$pairs, produced in photon-photon collisions, and their annihilation. Also, as noted by Niedźwiecki et al. (2016), relxill and relxilllp do not include the redshift of direct photons (only the reflected ones are redshifted), which represents a further problem for the lamppost model of P15. (Fortunately, this does not a major problem for our relxillcp fits, because the fitted values of the inner disc edge are relatively far from the $\mathrm{BH}$, in which case the redshift is small.) Furthermore, the size of the lamppost in the model of P15 has to be very small, and thus unable to intercept enough disc photons to Comptonise them (Dovciak \& Done 2016).

Another issue is that the theoretical spectrum from the lamppost fitted with the model of $\mathrm{P} 15$ has the reflection component more twice as strong as that observed. For the best fit of this model, the theoretical reflection strength is 0.41 , while we observe $0.18-0.20$ (Table 2). The way the lamppost fit was achieved in P15 was to allow the normalization of the reflected component to be free, and then reduced with respect to the actual lamppost geometry. This is a common practice in lamppost modelling, e.g, Keck et al. (2015), Fürst et al. (2015), but it is clearly not the correct procedure in the case of a specified geometry ${ }^{6}$.

On the other hand, the lamppost may be thought of as an approximation to a vertically stratified source, as discussed in P15. In this case, the observed direct power law can be emitted mostly higher up on the rotation axis, and have a negligible redshift. Still, in order to obtain the observed reflection component from the lamppost part close to the horizon, that part has to emit a strong Comptonization component. Then, the accretion rate has to account for photons swallowed by the $\mathrm{BH}$. This would still result in the accretion rate in the hard state of Cyg X-1 being larger than that in the

6 We note that the reflection strength is a free parameter in our fits of coronal models, as well as in other such studies in literature. This is justified by the geometry being not fully specified, e.g., in our case a hot inner flow overlapping with an outer disc, with the unknown amount of overlap and the scale height of the hot flow. 
soft state. Furthermore, an upper lamppost component, which dominates the observed power law in that scenario, would give rise to a strong, weakly-blurred, reflection component, and it is not clear whether a good fit could be obtained with such model.

The same problem occurs in our lamppost fit, Model 4. The theoretical reflection strength for it is 0.59 , while we observe three times less, 0.20 (Table 2). Since here the lamppost height is already about $10 R_{\mathrm{g}}$, a vertical stratification discussed above would not help. Thus, we consider this model not to be self-consistent.

\subsection{The inner disc radius}

As discussed above, we have found that although the model of the irradiating spectrum of P15 was valid as a phenomenological continuum, it was not physically self-consistent. That model yielded low values of the truncation radii, $r_{\text {in }} \simeq 2.5 \pm 0.2$. We have searched for physical models that would yield similar continua. Very similar, but physical, models feature two or more thermal Comptonization components. The models with two components consist of the standard hard thermal Comptonization component, and a soft one, representing the soft excess. Our best Model 3 yields $r_{\text {in }} \simeq 13-20$, and it is statistically preferable to that of P15. The specific concave shape of our model is responsible for a reduction of the red tail in the relativistic line profile. This effect has been known for long, probably starting with Woźniak et al. (1998), who found it for broad-line radio galaxies.

On the other hand, we have also found that the lamppost model with the hard thermal Comptonization continuum and an additional soft thermal continuum provides a reasonable fit to the data, and yields a low inner radius between the ISCO and $\simeq 7 R_{\mathrm{g}}$. The height of the lamppost is relatively large, $\simeq 10 R_{\mathrm{g}}$. However, as discussed above, the reflection strength in that model is three times higher as that observed.

We note that the relatively large confidence interval of the inner radius in this model is related to the large height of the lamppost. At this height, light bending is weak, and the source is almost isotropic in the observer's frame. Thus, the solid angle subtended by innermost parts of the disc is small. Consequently, the relativistic broadening of the line is moderate, and the line is significantly narrower than in the lamppost model of P15, at which the lamppost was almost on the horizon, and photons were strongly bent toward to the disc. The degeneracy between the lamppost height and the inner radius was pointed out by Fabian et al. (2014). In Fig. 4, we also see that there is degeneracy between this model and the coronal Model 1. The two look identical in spite of the different inner radii, which is due to the small contribution of the innermost radii to reflection in Model 4, with its large lamppost height.

Overall, we interpret our results as showing that the presence of a substantially truncated disc in Cyg X-1 is fully compatible with the data, as well as preferred both statistically and based on physical considerations, see Section 1. Still, Fig. 5, comparing the residuals of different models, shows that there are no clear systematic differences in the residual patterns. We feel that based on our data alone, we cannot exclude the existence of a physical model yielding a low truncation radius.

\section{CONCLUSIONS}

We have performed a detailed spectral study of simultaneous observations of Cyg X-1 in the hard state by NuSTAR and Suzaku. The same data were analysed before, and found to be well modelled by a lamppost located on the axis of a rotating BH very close to the horizon. The surrounding disc was found to extend close to the innermost stable circular orbit. We have found here that the model of the incident radiation used in P15 was not, unfortunately, physically self-consistent, although it was valid as a phenomenological continuum.

In the present study, we model the incident continuum by two thermal Comptonization components, which reproduce in a physically self-consistent and plausible way the broad-band spectrum of Cyg X-1 including a prominent soft excess present in this source. Our best model, together with Compton reflection of both components, implies the accretion disc is truncated at $\sim 13-20$ gravitational radii. However, we find that the derived inner radius in the hard state is dependent on the continuum model used, and further work is needed to explore physically-motivated multi-component Comptonization models.

\section{ACKNOWLEDGMENTS}

We thank John Tomsick for providing us with the wind-absorption model used in this work, and Thomas Dauser, Chris Done, Javier García, Andrzej Niedźwiecki, and Jörn Wilms for valuable discussions. This research has made use of data obtained through the HEASARC Online Service, provided by the NASA/GSFC, in support of NASA High Energy Astrophysics Programs. RB is a stipendiary of the 2016 START program of the Polish Science Foundation. This research has been supported in part by the Polish National Science Centre grants 2013/10/M/ST9/00729 and 2015/18/A/ST9/00746.

\section{REFERENCES}

Anders E., Grevesse N., 1989, GeCoA, 53, 197

Balucinska-Church M., Belloni T., Church M. J., Hasinger G., 1995, A\&A, 302, L5

Bałucińska-Church M., Church M. J., Charles P. A., Nagase F., LaSala J., Barnard R., 2000, MNRAS, 311, 861

Basak R., Zdziarski A. A., 2016, MNRAS, 458, 2199

Bautz M. W., et al., 2009, PASJ, 61, 1117

Beer M. E., Podsiadlowski P., 2002, MNRAS, 331, 351

Bowyer S., Byram E. T., Chubb T. A., Friedman H., 1965, Science, 147, 394

Coppi P. S., 1999, in Poutanen J., Svensson R., eds., ASP Conf. Ser. Vol. 161, High Energy Processes in Accreting Black Holes. Astron. Soc. Pac., San Francisco, p. 375

Dauser T., Wilms J., Reynolds C. S., Brenneman L. W., 2010, MNRAS, 409, 1534

De Marco B., Ponti G., 2016, ApJ, 826, 70

De Marco B., Ponti G., Muñoz-Darias T., Nandra K., 2015, ApJ, 814, 50

De Marco B., et al., 2017, MNRAS, submitted

Di Salvo T., Done C., Życki P. T., Burderi L., Robba N. R., 2001, ApJ, 547, 1024

Done C., Diaz Trigo M., 2010, MNRAS, 407, 2287

Done C., Zycki P. T., 1999, MNRAS, 305, 457

Done C., Gierliński M., Kubota A., 2007, A\&ARv, 15, 1

Dovciak M., Done C., 2015, arXiv:1507.05564

Dovciak M., Done C., 2016, Astronomische Nachrichten, 337, 441

Fabian A. C. et al., 2012, MNRAS, 424, 217

Fabian A. C., Parker M. L., Wilkins D. R., Miller J. M., Kara E., Reynolds C. S., Dauser T., 2014, MNRAS, 439, 2307

Frontera F. et al., 2001, ApJ, 546, 1027

Fürst F., et al., 2015, ApJ, 808, 122

García J., Kallman T. R., 2010, ApJ, 718, 695 
García J. et al., 2014, ApJ, 782, 76

Gierliński M., Zdziarski A. A., 2005, MNRAS, 363, 1349

Gierliński M., Zdziarski A. A., Done C., Johnson W. N., Ebisawa K., Ueda Y., Haardt F., Phlips B. F., 1997, MNRAS, 288, 958 1997, MNRAS, 288, 958

Gierliński M., Zdziarski A. A., Poutanen J., Coppi P. S., Ebisawa K., Johnson W. N., 1999, MNRAS, 309, 496

Gierliński M., Zdziarski A. A., Done C., 2011, in Frontier Objects in Astrophysics and Particle Physics, ed. F. Giovannelli and G. Mannocchi, Italian Physical Soc. Conf. Proc. 103, p. 299, arXiv:1011.5840

Gies D. R. et al., 2003, ApJ, 583, 424

Gilfanov M., 2010, in Belloni T. ed., The Jet Paradigm: From Microquasars to Quasars, Lecture Notes in Physics 794. Springer, Berlin, Heidelberg, p. 17

Gou L. et al., 2011, ApJ, 742, 85

Gruber D. E., Matteson J. L., Peterson L. E., Jung G. V., 1999, ApJ, 520, 124

Harrison F. A. et al., 2013, ApJ, 770, 103

Hjellming R. M., Rupen M. P., 1995, Nature, 375, 464

Hutchings J. B., 1976, ApJ, 203, 438

Ibragimov A., Poutanen J., Gilfanov M., Zdziarski A. A., Shrader C. R., 2005, MNRAS, 362, 1435

Ingram A., Done C., 2011, MNRAS, 415, 2323

Ingram A., Done C., Fragile P. C., 2009, MNRAS, 397, L101

Kallman T., Bautista M., 2001, ApJS, 133, 221

Kawano T., Done C., Yamada S., Takahashi H., Axelsson M., Fukazawa Y., 2017, PASJ, 69, 36

Keck M. L., et al., 2015, ApJ, 806, 149

Kolehmainen M., Done C., Díaz Trigo M., 2014, MNRAS, 437, 316

Körding E., Falcke H., 2004, A\&A, 414, 795

Kotov O., Churazov E., Gilfanov M., 2001, MNRAS, 327, 799

Koyama K. et al., 2007, PASJ, 59, 23

Lasota J.-P., Narayan R., Yi I., 1996, A\&A, 314, 813

Madsen K. K., et al., 2015, ApJS, 220, 8

Makishima K., Maejima Y., Mitsuda K., Bradt H. V., Remillard R. A., Tuohy I. R., Hoshi R., Nakagawa M., 1986, ApJ, 308, 635

Makishima K., et al., 2008, PASJ, 60, 585

Martocchia A., Matt G., 1996, MNRAS, 282, L53

McConnell M. L., et al., 2002, ApJ, 572, 984

Miller J. M., Homan J., Steeghs D., Rupen M., Hunstead R. W., Wijnands R., Charles P. A., Fabian A. C., 2006, ApJ, 653, 525

Miller J. M., Pooley G. G., Fabian A. C., Nowak M. A., Reis R. C., Cackett E. M., Pottschmidt K., Wilms J., 2012, ApJ, 757, 11

Mitsuda K. et al., 1984, PASJ, 36, 741

Mitsuda K. et al., 2007, PASJ, 59, 1

Narayan R., Yi I., 1995, ApJ, 452, 710

Niedźwiecki A., Zdziarski A. A., Szanecki M., 2016, ApJ, 821, L1

Novikov I. D., Thorne K. S., 1973, in Black Holes, eds. C. DeWitt and B. DeWitt, Gordon and Breach, Paris, p. 343

Nowak M. A. et al., 2011, ApJ, 728, 13

Orosz J. A., McClintock J. E., Aufdenberg J. P., Remillard R. A., Reid M. J., Narayan R., Gou L., 2011, ApJ, 742, 84

Ota N., et al., 2008, A\&A, 491, 363

Overbeck J. W., 1965, ApJ, 141, 864

Parker M. L., et al., 2015, ApJ, 808, 9 (P15)

Plant D. S., Fender R. P., Ponti G., Muñoz-Darias T., Coriat M., 2015, A\&A, 573, 120

Poutanen J., Coppi P. S., 1998, PhST, 77, 57

Poutanen J., Svensson R., 1996, ApJ, 470, 249

Poutanen J., Vurm I., 2009, ApJ, 690, L97

Poutanen J., Krolik J. H., Ryde F., 1997, MNRAS, 292, L21

Poutanen J., Zdziarski A. A., Ibragimov A., 2008, MNRAS, 389, 1427

Predehl P., Schmitt J. H. M. M., 1995, A\&A, 293, 889

Reid M. J., McClintock J. E., Narayan R., Gou L., Remillard R. A., Orosz J. A., 2011, ApJ, 742, 83

Reis R. C., Fabian A. C., Miller J. M., 2010, MNRAS, 402, 836

Revnivtsev M., Gilfanov M., Churazov E., 1999, A\&A, 347, L23
Rykoff E. S., Miller J. M., Steeghs D., Torres M. A. P., 2007, ApJ, 666, 1129

Shakura N. I., Sunyaev R. A., 1973, A\&A, 24, 337

Shapiro S. L., Lightman A. P., Eardley D. M., 1976, ApJ, 204, 187

Smith R. K., Valencic L. A., Corrales L., 2016, ApJ, 818, 143

Takahashi T. et al., 2007, PASJ, 59, 35

Titarchuk L., 1994, ApJ, 434, 570

Tomsick J. A. et al., 2008, ApJ, 680, 593

Tomsick J. A. et al., 2014, ApJ, 780, 78

Tsujimoto M., et al., 2011, A\&A, 525, A25

Walborn N. R., 1973, ApJ, 179, L123

Walton D. J., et al., 2016, ApJ, 826, 87

Woźniak P. R., Zdziarski A. A., Smith D., Madejski G. M., Johnson W. N., 1998, MNRAS, 299, 449

Wu Y. X., Li T. P., Belloni T. M., Wang T. S., Liu H., 2009, ApJ, 695, 921

Wu Y. X., Belloni T. M., Stella L., 2010, MNRAS, 408, 2413

Xiang J., Lee J. C., Nowak M. A., Wilms J., 2011, ApJ, 738, 78

Yamada S., Makishima K., Done C., Torii S., Noda H., Sakurai S., 2013, PASJ, 65,80

Yuan F., Narayan R., 2014, ARA\&A, 52, 529

Zdziarski A. A., Gierliński M., 2004, Prog. Theor. Phys. Suppl., 155, 99

Zdziarski A. A., Johnson W. N., Magdziarz P., 1996, MNRAS, 283, 193

Zdziarski A. A., Lubiński P., Smith D. A., 1999, MNRAS, 303, L11

Zdziarski A. A., Lubiński P., Gilfanov M., Revnivtsev M., 2003, MNRAS, 342,355

Ziółkowski J., 2014, MNRAS, 440, L61 